\title{
Split-thickness skin graft coverage following release of postburn first web space adduction contracture
}

\author{
Charles FT Snelling MD FRCSC, Eva Germann MSc \\ Division of Plastic Surgery, Vancouver Hospital and Health Sciences Centre, University of British \\ Columbia, Vancouver, British Columbia
}

\begin{abstract}
CFT Snelling, E Germann. Split-thickness skin graft coverage following release of postburn first web space adduction contracture. Can J Plast Surg 1999;7(5):219-227.

Twelve patients from 11 to 57 years of age who underwent surgical release of postburn first web space thumb adduction-pronation contracture had the resulting defect resurfaced successfully with a medium-thickness, unmeshed, split-thickness skin graft (STSG). This procedure provided satisfactory, durable cover and maintained the gain in thumb abduction. All hands had combined dorsal and volar scarring that required incision. If indicated, partial division of the midbelly of the distal border of the adductor and distal midborder of the first dorsal interosseous was performed to increase both radial and palmar thumb abduction. Immediate or delayed skin grafting, the latter with a dynamic grafting splint, was carried out. Fifteen hands (from 12 patients) were assessed one year or more after surgical release. The mean postoperative radial abduction angle measured on photographs between the first metacarpal shaft and index proximal phalanx was $42^{\circ} \pm 13^{\circ}$, which was greater than the preoperative mean of $23^{\circ} \pm 12^{\circ}(\mathrm{P}=0.001)$ but less than the mean of $57^{\circ} \pm 12^{\circ}$ for 20 nonburned control subjects $(\mathrm{P}=0.002)$. The mean postoperative palmar abduction angle measured between the first metacarpal and the proximal phalanx of the index finger was $54^{\circ} \pm 19^{\circ}$, which was greater than the preoperative mean of $18^{\circ} \pm 21^{\circ}(\mathrm{P}<0.001)$. An STSG was simple and quick to harvest for the burned hand at or near full growth size, and was successful in maintaining the increased abduction gained in the first web space for most of the patients who were treated.
\end{abstract}

Key Words: Burn hand; Burn scar; First web space contracture; Hand reconstruction; Skin graft; Thumb adduction contracture

\section{Revêtement par greffe de peau demi-épaisse après la libération d'une rétraction en adduction de la première commissure du pouce survenue après une brûlure}

RÉSUMÉ : Douze patients âgés de 11 à 57 ans chez qui on a procédé à une libération chirurgicale d'une rétraction en adduction-pronation de la première commissure du pouce survenue après une brûlure ont, pour cacher les cicatrices résultant de cette intervention, subi avec succès un resurfaçage avec une greffe de peau demi-épaisse, sans filets, d'épaisseur moyenne. Cette intervention a fourni un revêtement durable et satisfaisant et maintenu l'augmentation de l'adduction du pouce. Toutes les mains avaient une combinaison de cicatrices antérieures et dorsales qui ont nécessité une incision. Lorsqu'il y avait indication, une division partielle du corps moyen du bord distal de l'adducteur et du bord moyen distal du premier interosseux dorsal a été réalisée pour augmenter à la fois l'abduction palmaire et radiale du pouce. On a ensuite procédé à une greffe de peau immédiate ou tardive, dans ce dernier cas, par la pose d'un greffon maintenu par une attelle dynamique. Quinze mains (de 12 patients) ont été évaluées un an et plus après la libération chirurgicale. L'angle moyen de l'abduction radiale postopératoire mesuré sur des photographies entre le corps du premier métacarpien et la phalange proximale de l'index était de $42^{\circ} \pm 13^{\circ}$, ce qui était supérieur à la moyenne préopératoire de $23^{\circ} \pm 12^{\circ}(\mathrm{p}=0,001)$, mais moins que la moyenne de $57^{\circ} \pm 12^{\circ}$ pour les 20 sujets témoins non brûlés $(\mathrm{p}=0,002)$. L'angle moyen de l'abduction palmaire postopératoire mesuré entre le premier métacarpien et la phalange proximale de l'index était de $54^{\circ} \pm 19^{\circ}$, ce qui était supérieur que la moyenne préopératoire de $18^{\circ} \pm 21^{\circ}$ (p $<0,001$ ). Une greffe de peau demi-épaisse s'est avérée simple et rapide à prélever pour la main brûlée à une taille de croissance complète ou presque complète, et a pu garantir l'augmentation de l'abduction gagnée dans la première commissure pour la plupart des patients traités.

This paper was presented to the Northwest Plastic Surgery Society, January 31, 1996, Whistler, British Columbia and, in part, at the 45th Annual Meeting of the Canadian Society of Plastic Surgeons, June 27, 1991, Whistler, British Columbia

Correspondence: Dr C Snelling, \#306-888 W 8th Avenue, Vancouver, British Columbia V5Z 3Y1. Telephone 604-873-9333, fax 604-874-6717, e-mailsnelling@interchange.ubc.ca

\begin{abstract}
A dduction contraction of the first web space following burn injury can disable the hand by reducing the thumbindex span, limiting the size of objects that can be grasped. Severe proximal palmar scarring can produce transverse cupping, and full flexion of the index and long fingers may be blocked by the thumb if it is permanently pronated across the palm.
\end{abstract}




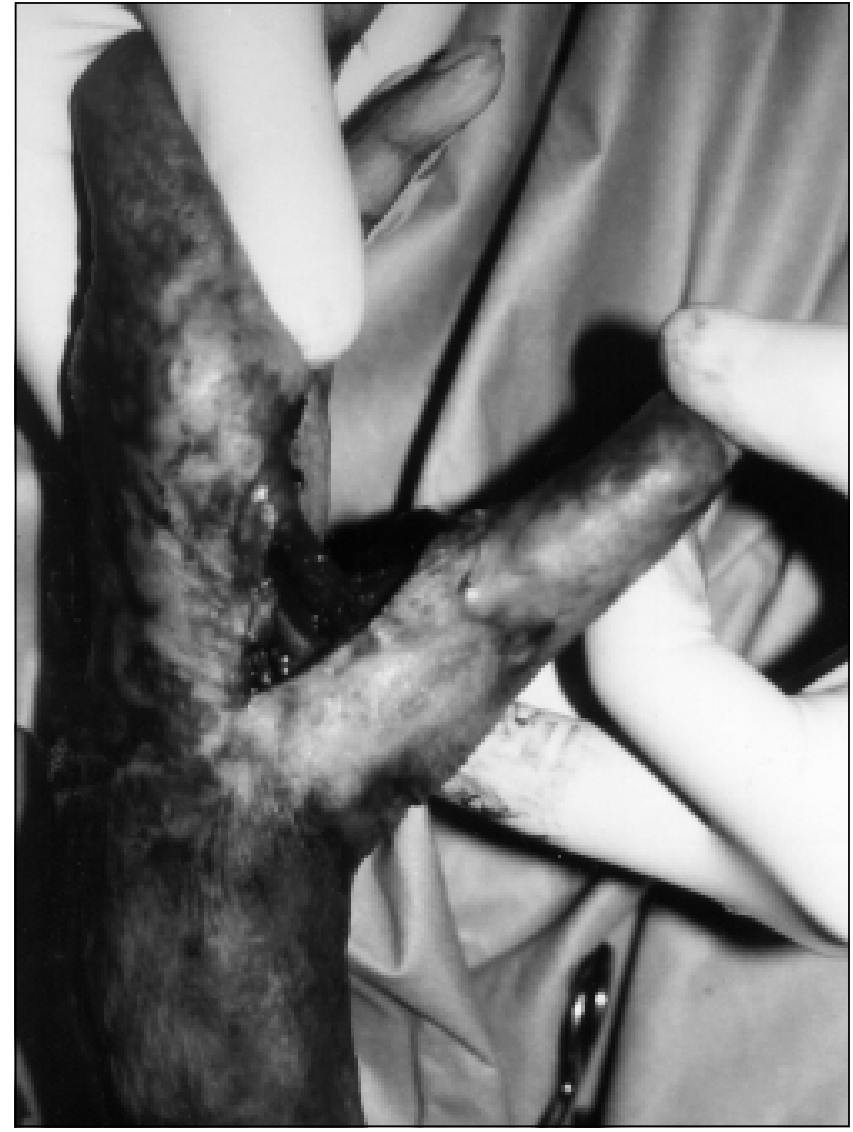

Figure 1) Intraoperative photo of patient $12 \mathrm{~b}$ showing proximal extent of the dorsal incision and division border of the first metacarpal origin of the first dorsal interosseous

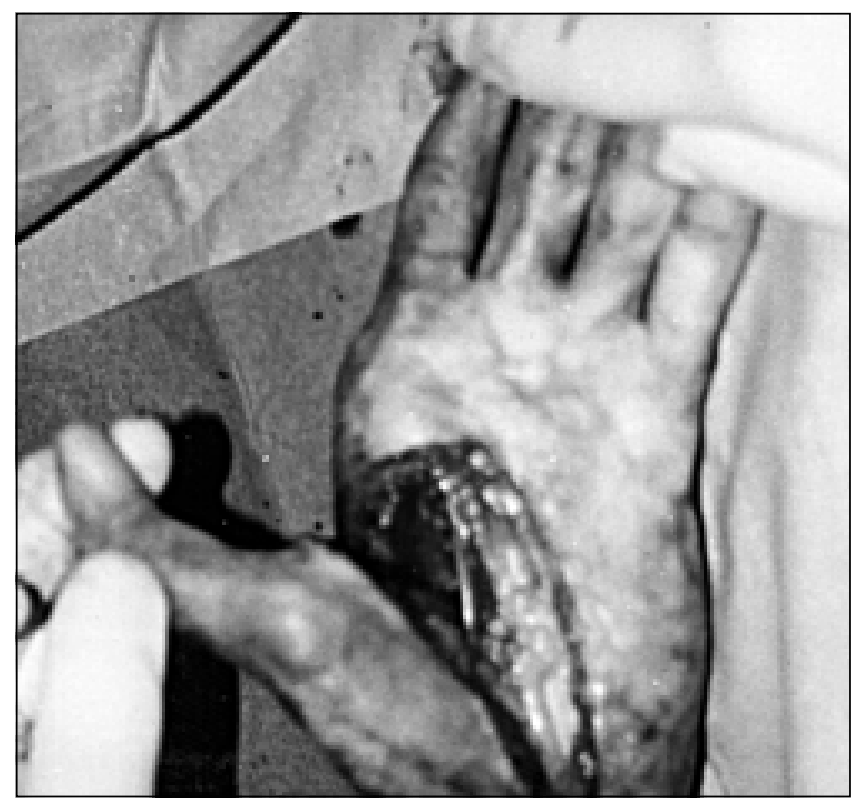

Figure 2) Intraoperative photo of patient $12 \mathrm{~b}$ showing the extent of palmar release incision of first web extending proximally about the thenar eminence to include a carpal tunnel release. Pronation has been further released by dividing the midportion of the distal border of the adductor. No scar tissue was excised, and the raw area was created entirely by incision

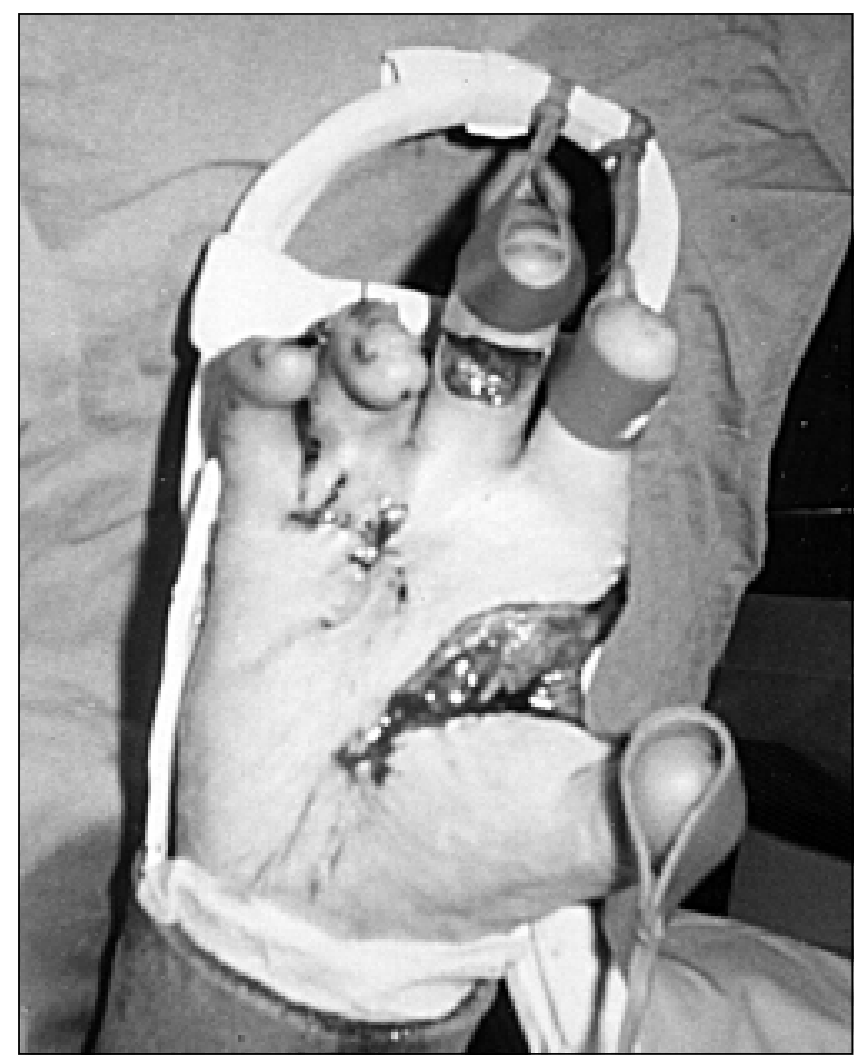

Figure 3) Intraoperative photo of patient 6 a following release showing the right hand in a dynamic grafting splint with outriggers positioning the hand in position achieved by release before application of the splitthickness skin graft, which was delayed $24 \mathrm{~h}$. Note the splint was constructed to permit easy access to the graft sites. The proximal interphalangeal joint of the long finger was also released and grafted

Release of the first web space contracture, which may require division of part of the first dorsal interosseous and/or adductor, can leave a significant skin defect, which has been resurfaced by the full spectrum of methods from split-thickness and full-thickness grafts to local and distant pedicle and free flaps. Fifteen hands from twelve patients with first web space contracture were treated by a soft-tissue release and coverage with medium-thickness sheet split-thickness skin grafts (STSGs). The technique using delayed skin application with the hand positioned in a dynamic grafting splint has been described previously by Snelling (1). The present paper reviews the outcome results in 15 hands evaluated at least 12 months after release.

\section{Surgical technique}

\section{PATIENTS AND METHODS}

The surgical release is carried out under tourniquet control following exsanguination. The incision starts at the midpoint of the web between the thumb and index fingers. On the dorsal surface, the incision runs proximally toward the junction of the first and second metacarpal bases, exposing the first dorsal interosseous (Figure 1). On the palmar surface, the in- 


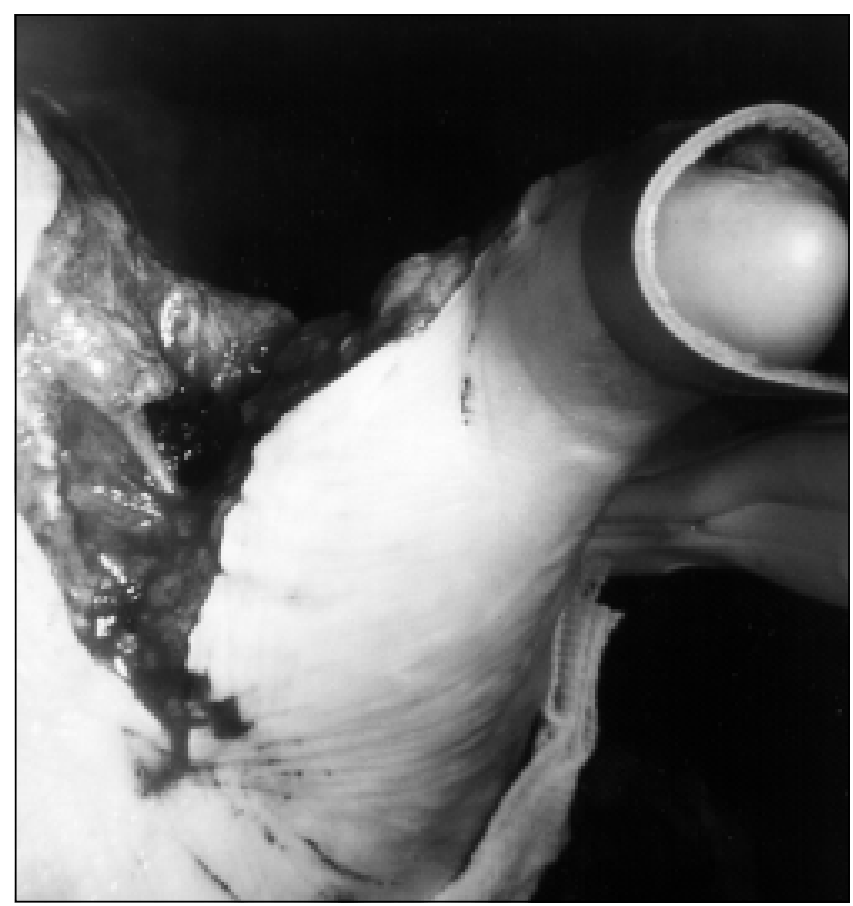

Figure 4) Palmar view of right hand web of patient 6 a with palm up, thumb to the right, index to the left and dorsum down in contact with the splint. On the volar surface, the incision through the distal border of the adductor is seen, which is above and to the right of the incision through the distal border of the portion of the first dorsal interosseous arising from the first metacarpal, which is below and left relative to the adductor incision

cision courses ulnarly and then proximally to the distal ulnar border of the thenar eminence (Figure 2). Sharp dissection is used through the skin and scar tissue, but as the deep surface of the scar tissue is approached, blunt dissection is employed, which is aided by alternating palmar and radial abduction of the thumb ray. On the volar surface, the palmar fascia is encountered, and deep to this the neurovascular bundles to the radial side of the index and both sides of the thumb can be identified and protected (Figures 2,3). The adductor is identified on the palmar side deep to and between the neurovascular bundles. If adequate abduction in both radial and palmar planes is not possible, two further steps are taken: the distal border of the adductor is divided at the midpoint between the first and third metacarpal, leaving sufficient adductor muscle on the first metacarpal side to protect the bone and provide a bed for the skin graft (Figures 4,5), and the radial half of the first dorsal interosseous is divided well ulnar from the first metacarpal (Figures 1,4,5). Following this release, the neurovascular bundles to the radial side of the index finger and the thumb may bowstring slightly. If cupping between the thenar and hypothenar eminence remains, the skin incision is extended proximally, ulnar and parallel to the border of the thenar eminence toward the wrist crease directly over the carpal tunnel. If there is a thick

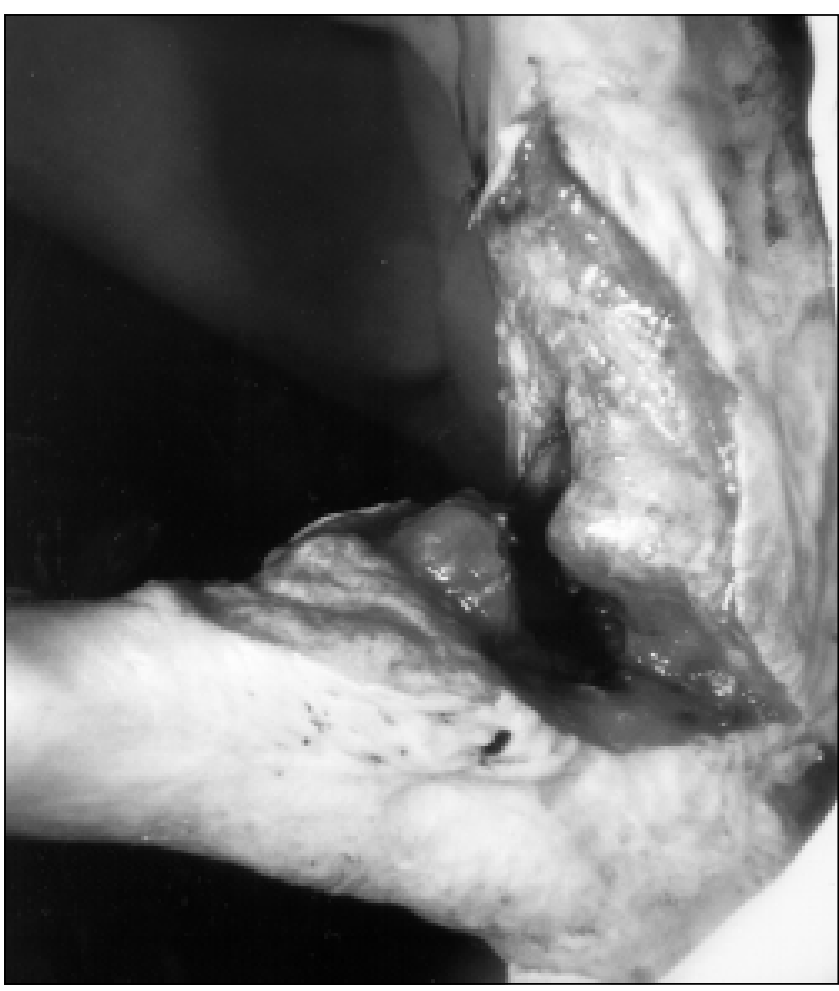

Figure 5) Dorsal view of patient 6 a with thumb to the left showing the division of the first dorsal interosseous close to the camera (dorsally), and the divided adductor behind (volar) and to the right

deep proximal palmar band bridging the thenar-hypothenar interspace, the carpal tunnel may also have to be opened, exposing the median nerve (Figure 2). The defect created by this extensive release leaves an irregular bed with a deep crevice between the first dorsal interosseous and the adductor, and over the carpal tunnel.

A medium-thickness STSG approximately $0.375 \mathrm{~mm}$ $(15 / 1000$ of an inch) thick is harvested. The release can be maintained by applying a preformed dynamic splint that will extend the finger metatarsophalangeal (MP) and interphalangeal (IP) joints, and radially and palmarly abduct the thumb with outriggers positioned to avoid contact with the raw defects (Figure 3). This splinting technique with delayed application of grafts after $24 \mathrm{~h}$ described previously by Snelling (1) was used for the first eight hands. The open-grafting technique involves a major postoperative nursing time commitment. For the last seven hands treated, immediate graft application was carried out in the operating room immediately following release. If immediate grafting is done, the tourniquet is deflated following the surgical release, major bleeding is identified and coagulated, and the tourniquet is reinflated before application of skin grafts.

A medium-thickness STSG $0.375 \mathrm{~mm}$ ( 15/1000 of an inch) thick is applied as a sheet and is cut considerably larger 


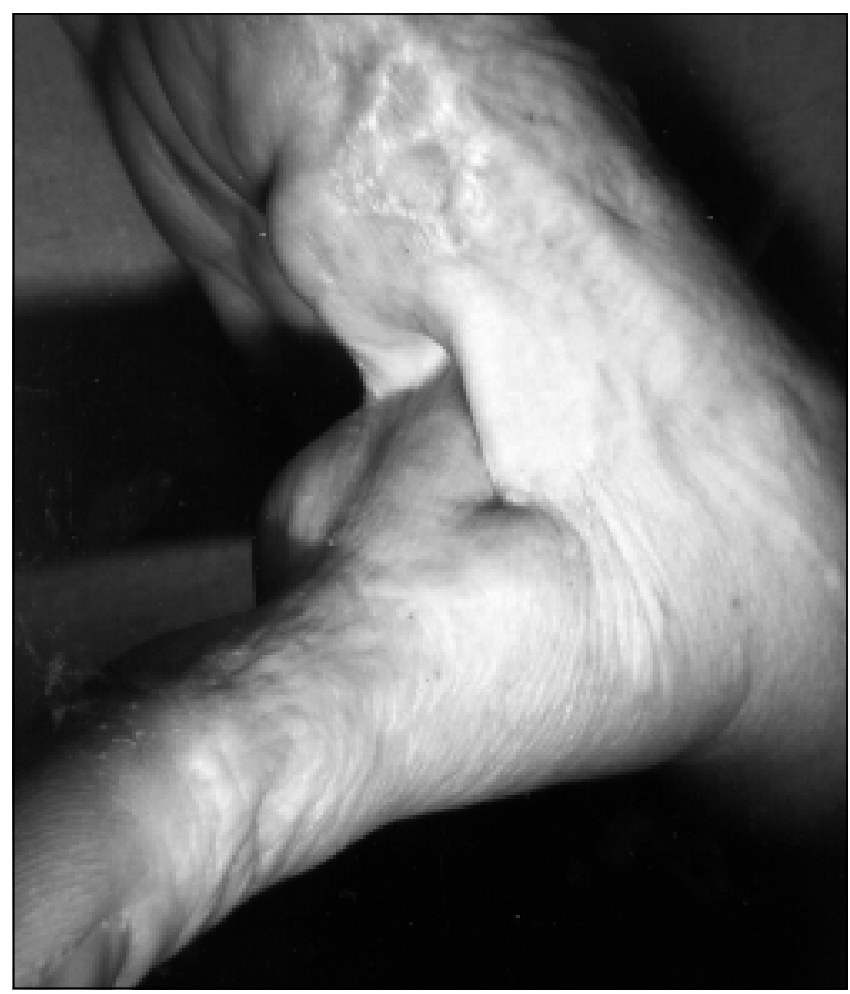

Figure 6) Postoperative result of patient 9 showing the same projection as the patient in Figure 5 - grafted cleft dorsal interosseous close to the camera, grafted adductor with the cleft behind and to the right, and graft in deep furrow between two muscles. Muscle skeletonization has not produced problems with postoperative strength

than the defect in order to allow it to drape and conform to all the irregular contours of the first web space. It must also be sufficiently large to overlap onto the intact skin so that shifting during application of the dressing will not expose the raw recipient bed of the margin. It must be draped into the crevice between the adductor and the first dorsal interosseous, and into the incision (Figures 4-6). Before application, a few pie-shaped incisions are made in the graft to eliminate air pockets and to promote adherence. Sheet graft was used because it was impossible to cover all recesses of this irregular wound with a meshed graft without expanding, which would expose the bed. The muscle is an excellent recipient site for STSG. Bowstringing of the neurovascular structures in the palm did not affect graft take and was not apparent after the graft matured. For immediate grafting, a well-moulded, conforming, liquid petroleum-soaked cotton wool dressing was applied to push the graft against all contours and to fill all cavities followed with a coarse, mesh gauze bolus dressing with enough bulk to completely fill the space between the thumb and index finger, and maintain the spread. For immobilization, the thumb was positioned midway between $90^{\circ}$ palmar abduction and $90^{\circ}$ radial abduction. Peripheral sutures inserted about the graft before the dressing was applied were tied over the bolus for compression.
Most patients treated with a delayed application had the graft left open, and it was inspected every $2 \mathrm{~h}$ to aspirate serum or blood that had accumulated below it. Where the dynamic splint had been used with open grafting, the finger and thumb stalls were removed periodically, and partial movement was started on postoperative day 2 . If occlusive dressings were used, these were soaked off on the fifth postoperative day. With both methods, a resting static splint worn periodically during the day and all night is important for the first two weeks. Elastic compression gloves were worn beginning two weeks after surgery and were supplemented with a moulded insert applied to the first web space inside the glove. The patient wore the glove and inserts at all times. After two weeks, a static C-shaped first web space splint was worn only at night for the next three months, and use of the hand was encouraged during the day.

\section{Assessment \\ Patients who underwent STSG coverage following release of postburn first web space adduction contracture were assessed a minimum of one year after release, with some assessed up to 10 years after reconstruction.}

Gain in thumb abduction - Radial abduction: Photographs of the hands with the palm up, fingers extended and the thumb fully extended and radially abducted parallel to the palmar plane were evaluated. A line was drawn parallel to the radial border of the thumb metacarpal (M1) and, a line drawn parallel to the radial border of the index proximal phalanx (P2). The radial abduction angle was the angle formed by the intersection of these two lines (M1 P2 angle).

Palmar abduction: On lateral photographs of the radial aspect of the hand and forearm with the fingers fully extended and the thumb abducted $90^{\circ}$ to the plane of the hand, a line was drawn parallel to the radial border of M1. Lines were drawn parallel to the dorsal border of M2 and parallel to the volar aspect of $\mathrm{P} 2$. The angles between the first metacarpal M1 and the index metacarpal M2 (M1 M2 angle), and between the first metacarpal M1 and the proximal phalanx of the index finger P2 (M1 P2 angle) were measured.

Controls: Adult subjects with normal hands were selected to measure the abduction angle between the thumb and index ray. Photographs of the right hand of each control were measured.

Statistical analysis: The preoperative and postoperative means and SD values of these three angles were determined and compared by paired Student's $t$ test. The means of these three angles for the 20 controls were compared with the postoperative means of the treated patients by Student's $t$ test. The null hypothesis was that there would be no difference between the control and the postoperative measurements (two-sided test) and that the preoperative means would be less than the postoperative values (one-sided test). 
TABLE 1

Demographic data of treated hands

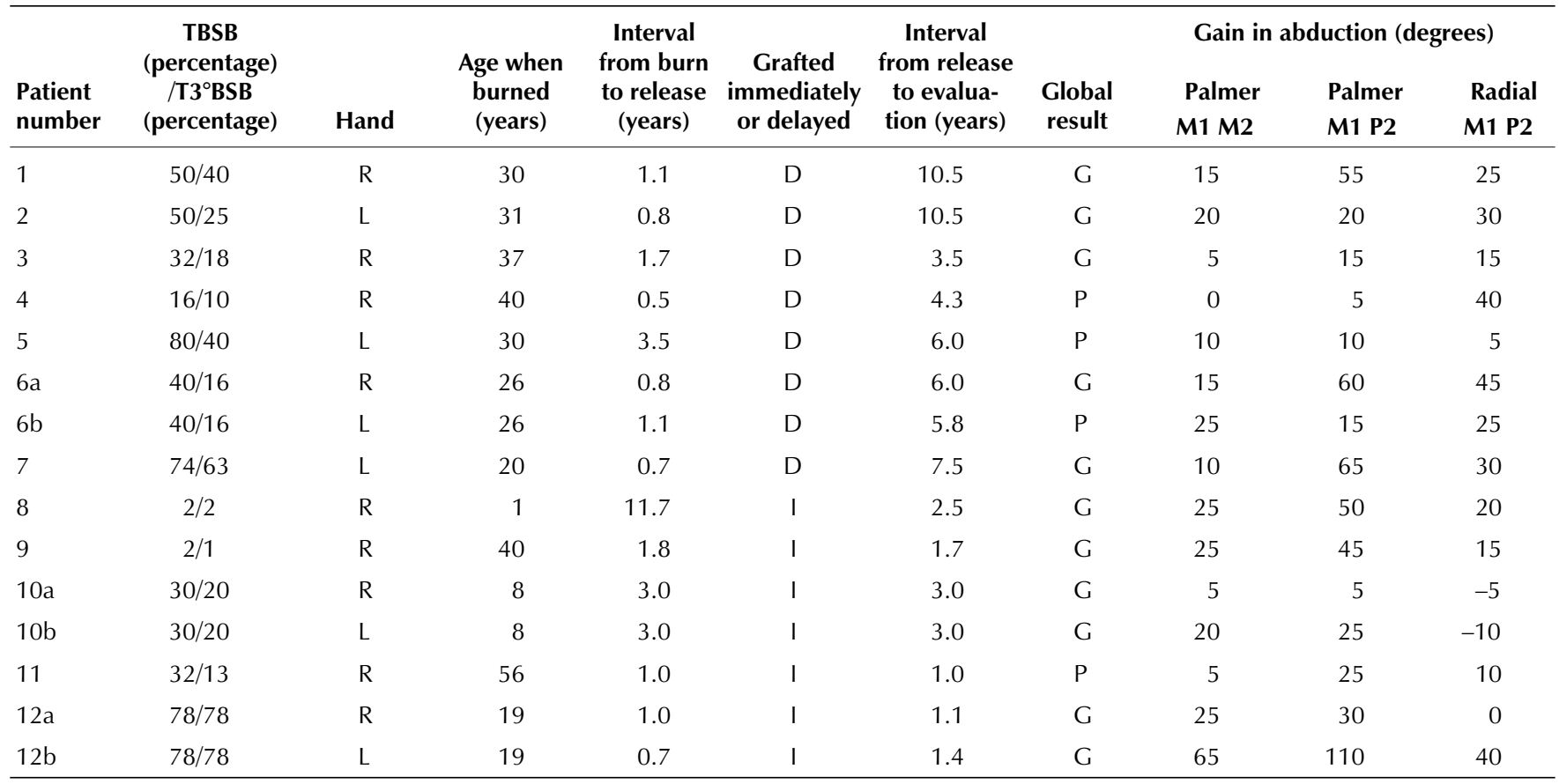

D Delayed; G Good; I Immediate; L Left; P Poor; R Right; TBSB Total body surface burn; T3BSB Total full-thickness body surface burn

TABLE 2

Comparison of preoperative and postoperative angles to each other and to controls

\begin{tabular}{|c|c|c|c|c|c|}
\hline & $\begin{array}{l}\text { Preoperative angle }\left(^{\circ}\right) \\
n=15\end{array}$ & $\mathbf{P}^{*}$ & $\begin{array}{l}\text { Postoperative angle }\left({ }^{\circ}\right) \\
n=15\end{array}$ & $\mathbf{P}^{+}$ & $\begin{array}{c}\text { Controls } \\
n=20\end{array}$ \\
\hline \multicolumn{6}{|c|}{ Palmar abduction } \\
\hline Mean \pm SD & $21 \pm 13$ & 0.001 & $39 \pm 12$ & 0.204 & $44 \pm 8$ \\
\hline Range & -5 to 45 & & 15 to 65 & & 30 to 60 \\
\hline Mean \pm SD & $18 \pm 21$ & 0.000 & $54 \pm 19$ & 0.055 & $65 \pm 11$ \\
\hline Range & -25 to 50 & & 25 to 85 & & 45 to 90 \\
\hline \multicolumn{6}{|l|}{ Radial abduction } \\
\hline \multicolumn{6}{|l|}{ M1 P2 angle } \\
\hline
\end{tabular}

*Paired Student's t test (preoperative versus postoperative). 'Student's t test (postoperative versus controls)

\section{Outcome}

The incidence of graft loss requiring regrafting and digital or palmar nerve injury with anesthesia or paresthesia postrelease was determined. Stability of the skin after the patient returned to full activity and a level of manual activity postoperatively comparable to preinjury activity and work were determined.

\section{Patient Data}

Twelve patients were evaluated (Table 1). Three patients had both hands treated. The mean age at the time of burn was 28 years, and the mean age at the time of the first web release was 31 years. Release was performed at a mean of 29 months postburn. One patient had both hands released at the same time (patient 10). 


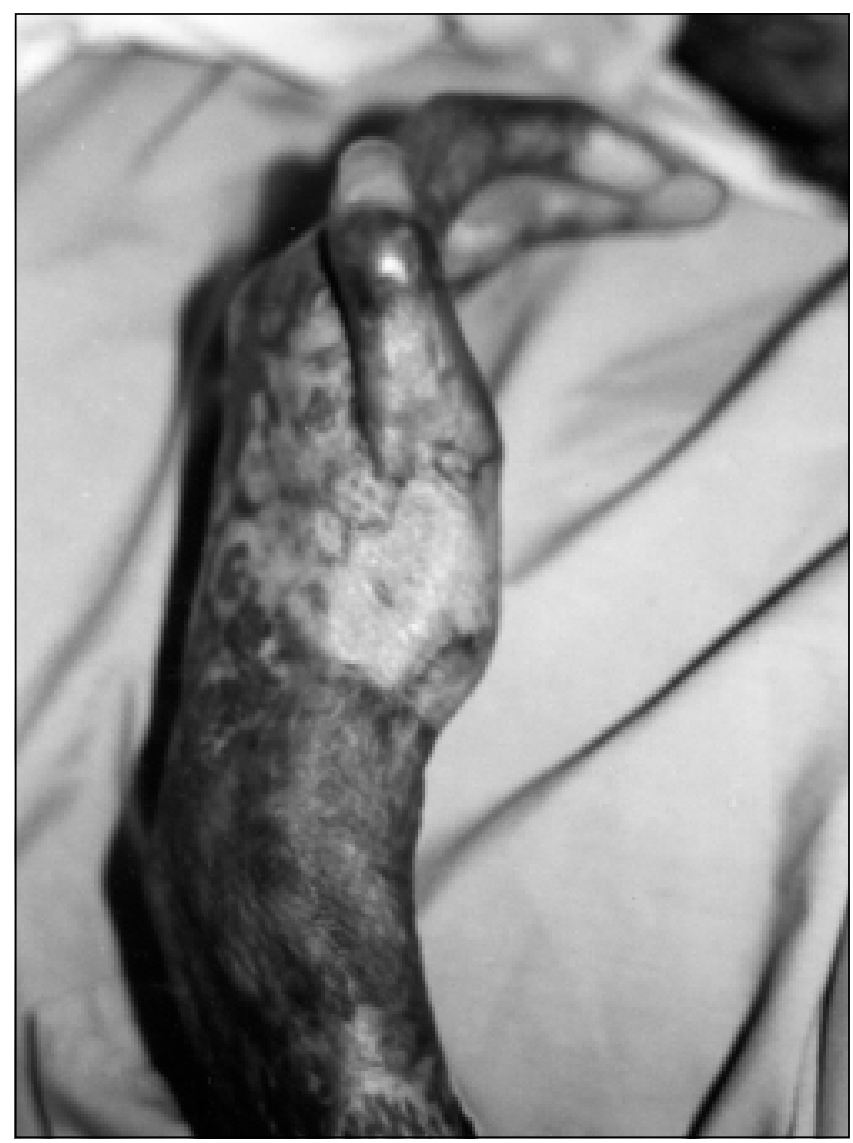

Figure 7) Patient 12 b eight months postburn showing palmar abduction preoperatively - Thumb metacarpal (M1) and index metacarpal (M2) parallel (M1 M2 angle zero, M1 P2 -25 $)$

\section{Technical details}

In the early part of the study period, grafting was delayed to the day following release in eight hands (patients 1 to 7) (Table 1). More recently, release was followed by immediate grafting of seven hands (patients 8 to 12). Release of the second, third or fourth web spaces was also combined with the first web space release in a few patients. Seven patients had concomitant digital volar flexion contractures corrected at same time.

\section{Difference in first web space angles preoperative to post-} operative-Palmar abduction: The preoperative M1 M2 angle was less than the postoperative angle $(\mathrm{P}<0.01)$ (Table 2$)$. Preoperative angles ranged from $-5^{\circ}$ where the first and second metacarpals were essentially parallel (Figure 7) up to $+45^{\circ}$, while postoperative ranges were from $+15^{\circ}$ to $+65^{\circ}$ (Figure 8). Similarly, the mean preoperative M1 P2 angle was less than the postoperative angle $(\mathrm{P}<0.01)$, with a much greater range in the preoperative from $-25^{\circ}$, a configuration in which the second (index) MP joint was flexed such that the M1 P2 lines would intersect distally rather than proximally to $+50^{\circ}$ (Figure 7). The postoperative range was $+25^{\circ}$ to $+85^{\circ}$ (Figure 8). Release in these cases, in addition to correcting

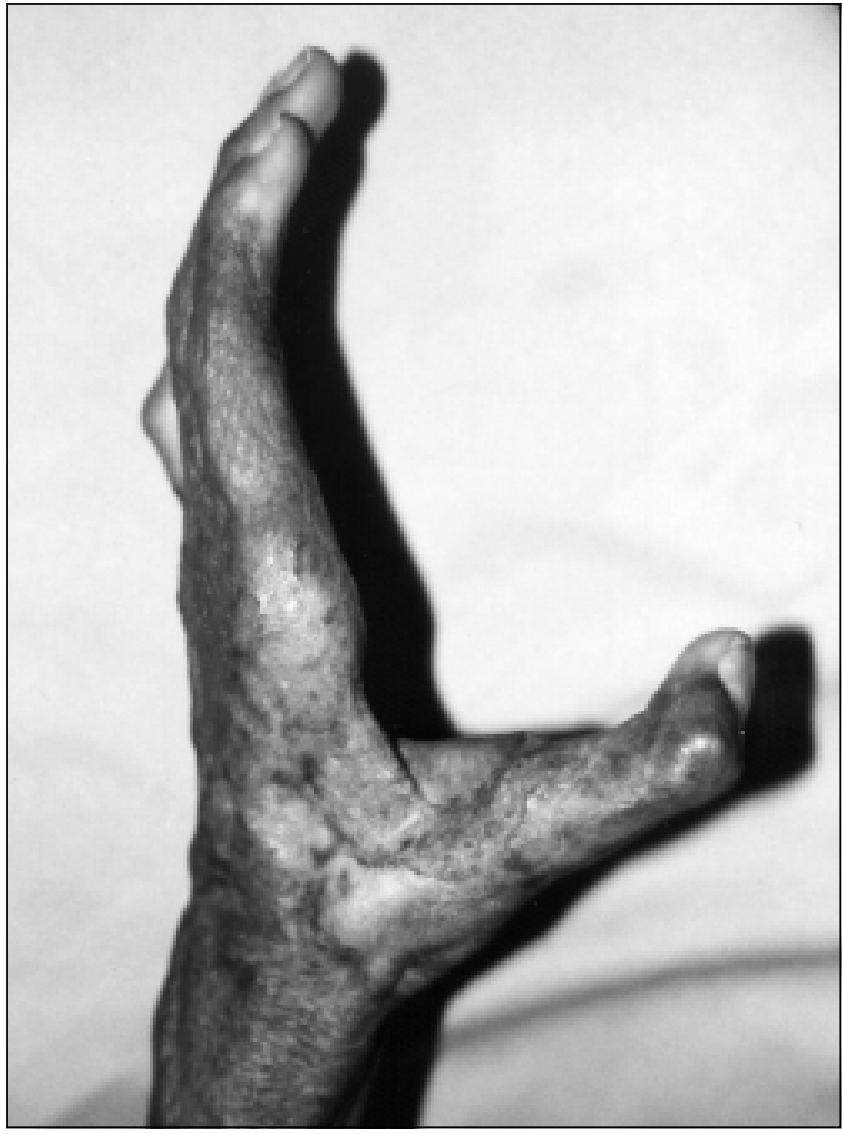

Figure 8) Patient $12 \mathrm{~b}$ at 17 months postrelease showing a good result. Palmar abduction gain of $65^{\circ}$ at the M1 M2 intersection and $110^{\circ}$ at the M1 P2 intersection (the patient was grafted immediately and closed). Note the skeletonized first dorsal interosseous (dorsal) and adductor behind (volar)

thumb adduction, also extended the scarred, flexed index MP joint. The M1 P2 angle measures both thumb abduction and second MP extension, but this is relevant because the second MP joint flexion contracture restricts thumb index separation restricting abduction.

Radial abduction M1 P2 angle: The preoperative M1 P2 mean angle was less than the postoperative angle $(\mathrm{P}<0.01)$ (Table 2). The range preoperatively was from $0^{\circ}$ to $+50^{\circ}$. Low preoperative angle values of $+10^{\circ}$ to $+15^{\circ}$ indicate that the first and second rays were almost parallel, and often indicated that the thumb was pronated across the palm, blocking index and middle finger flexion (Figure 9). Postoperative angles ranged from $+15^{\circ}$ to $+60^{\circ}$ (Figure 10) .

\section{Treated hands compared with controls}

In the control group, the mean of each of the three angles measured was greater than the corresponding preoperative mean (Table 2). The patients' postoperative mean M1 M2 palmar abduction angle was less, but did not differ significantly from the M1 M2 mean of controls. However, the patients' postoperative mean M1 P2 palmar abduction angle and 


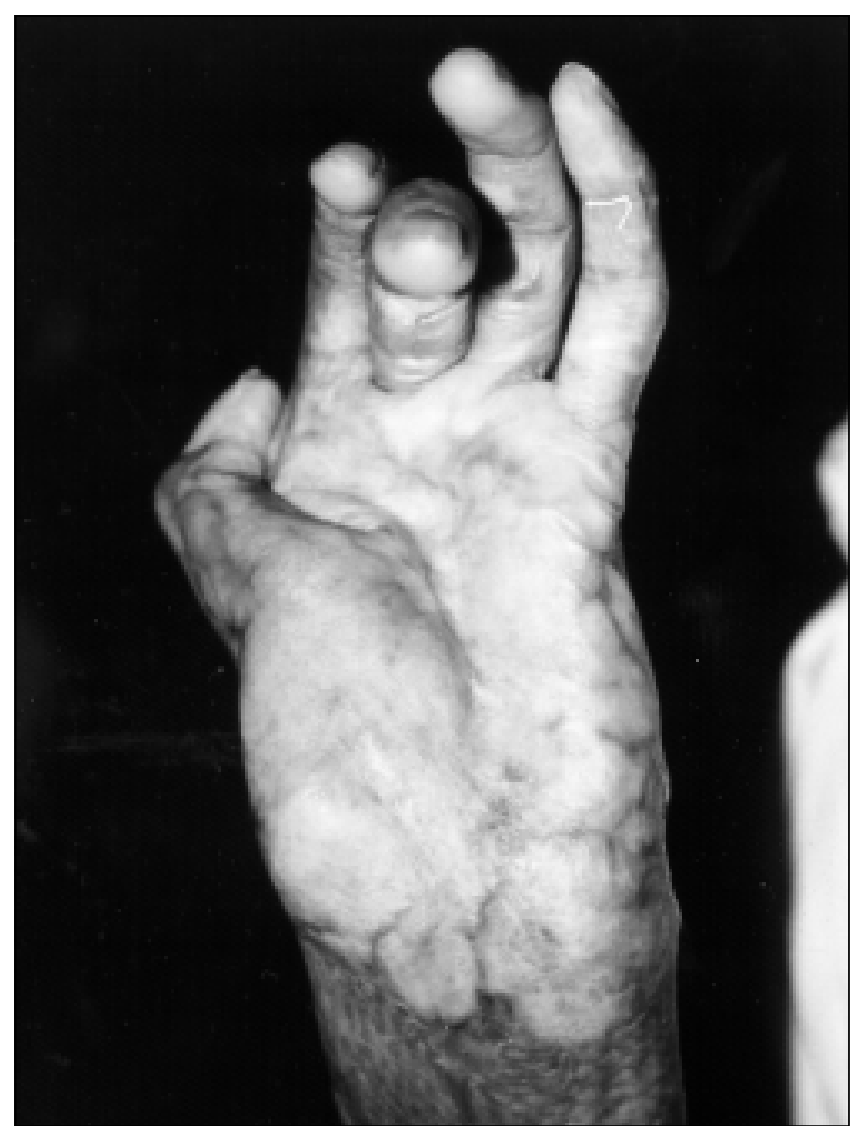

Figure 9) Patient $12 b$ at eight months postburn. Radial abduction preoperatively with thumb metacarpal (M1) and index metacarpal (P2) lines parallel. M1 was adducted and pronated across the palm, blocking the path of flexion of index and long fingers into the palm

postoperative mean M1 P2 radial abduction angle were significantly less than the corresponding means of the control hands, suggesting that the release obtained did not equal the spread of an unburned hand.

\section{Other findings}

There was no instance of graft failure, which is surprising considering that the grafted bed was quite irregular. Hematoma did not form below the graft in any case. Because the graft applied was larger than the defect draping over the peripheral intact skin, shifting, exposing the raw bed, did not occur.

One of the patients whose first web space release included proximal extension with concomitant carpal tunnel release had the palmar cutaneous branch of the median nerve transected, resulting in a painful neuroma at the volar wrist crease. There were no cases of digital nerve injury producing anesthesia or paresthesia of the thumb, index or radial side of the long finger, although skin grafts often had been applied directly to bared digital nerves. No patient reported breakdown of the palmar skin graft upon return to normal activity.

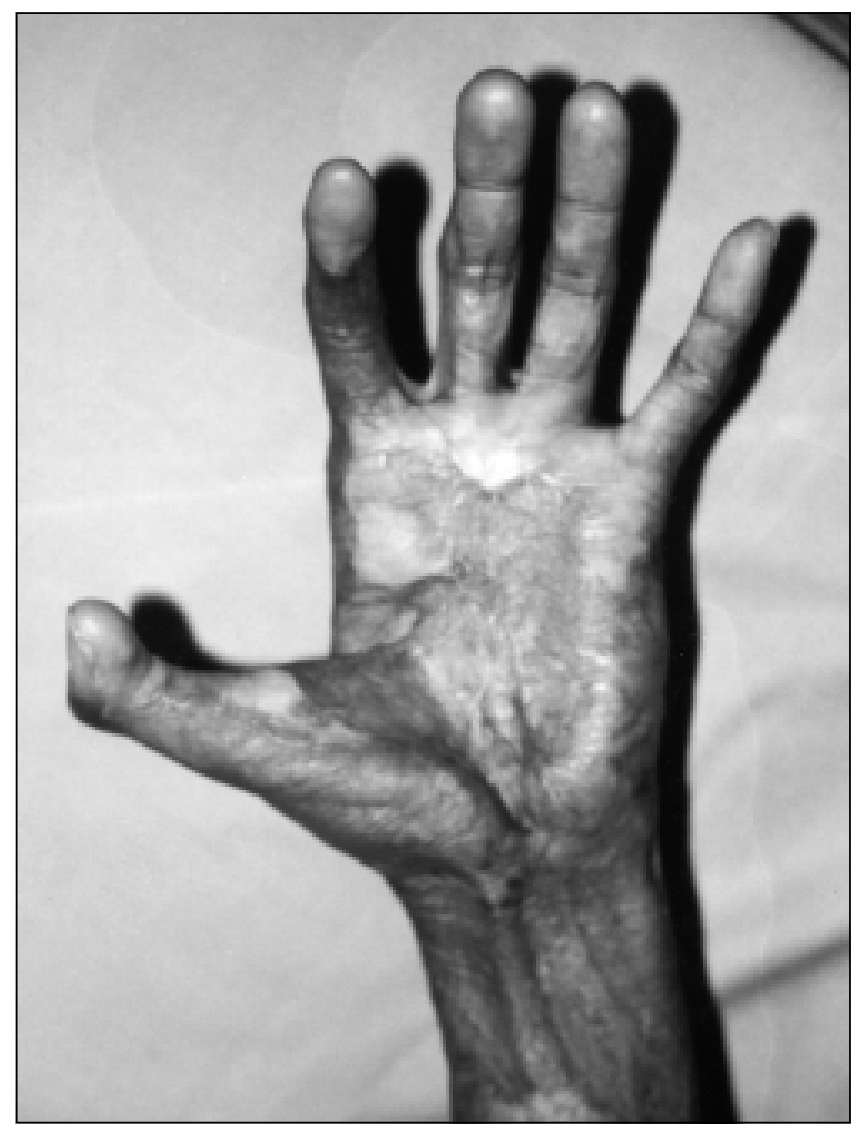

Figure 10) Patient $12 b$ at 17 months postrelease showing a good result. Radial abduction angle $M 1 P 2$ gain $40^{\circ}$. A skeletonized adductor can be seen

\section{Return to activity}

The outcome parameter 'return to activity' must be considered in the context that patients were evaluated from one year up to 10.5 years postrelease. Of the three patients who underwent bilateral releases, two were students and both have returned to full activity, while the third patient, who was unemployed at the time of burn, is now a mechanic.

Of the nine patients with unilateral release, two who were employed preoperatively are now unemployed, while the remaining seven are at the same or an equivalent job. One patient who is a carpenter is having difficulty with repeated wound breakdown of the dorsal surface of deeply burned MP and proximal interphalangeal joints but not the first web space. In many cases, the ability to use the hand was not determined solely by the success of the first web space release but by the total hand disability.

\section{DISCUSSION}

First web space contracture correction requires adequate release, permanent durable resurfacing and appropriate postoperative splinting until secondary wound contraction has 
ceased. In 12 patients eight years of age or older, resurfacing with a split-thickness graft provided durable closure and maintained the release, while requiring minimal time to harvest and minimal donor site morbidity compared with more complex closure methods. None required a bone block or internal spring device to maintain the abduction between the first and second metacarpal.

First web scar release with division of all or part of the first dorsal interosseous and/or adductor was described by Littler (2) to abduct the thumb following ischemic contracture of intrinsic muscles. Mutz (3) felt that the adductor was the main problem and divided its origin at the third metacarpal while preserving its first ray insertion. In the present series, partial incision in the middle of the two muscle bellies did not diminish postoperative thumb adduction power while providing an excellent bed to accept a graft.

Stern et al (4) emphasized the importance of evaluating both components of thumb abduction: namely, radial where the thumb is supinated and fully extended, and palmar where the thumb is at a $90^{\circ}$ angle to the plane of the palm because both were not always equally restricted preoperatively. Photographs were taken of all hands both palm up with the thumb abducted and extended in the plane of the palm, and from the radial side of the hand with the thumb palmarly abducted. Acknowledging that rotation of the hand with the camera at right angles to the hand can occur, a value of $\mathrm{P}<0.01$ rather than $\mathrm{P}<0.05$ between the mean angles compared was considered significant.

Stern et al (4) reviewed 102 burned hands in children with thumb and first web deformities that were characterized by one of four patterns. Palmar adduction deficit was identified in 67 patients. Of these patients, two-thirds were treated by a release and resurfacing with either split-thickness or fullthickness grafts, while the remainder were closed with Z-plasties or other local flaps. Opposition adduction deficit in which the thumb was pulled across the palm was described in an additional 25 patients of which 23 required a skin graft. Only two hands required release of the thumb adductor. It is unfortunate that these authors did not compare hands resurfaced with STSGs with those covered with full-thickness grafts.

Pensler et al (5) reviewed the cases of 25 children with deep partial-thickness palmar burns that initially reepithelialized but required secondary reconstruction for extensive palmar contractures that included adduction of the thumb and cupping between the thenar and hypothenar eminence. Following release, 11 received full-thickness skin grafts to the palm and 14 had STSGs. No difference was found after comparing the mean number of operations and final function between two groups, suggesting that either graft provided comparable long term function.

Schwanholt et al (6) compared split-thickness with fullthickness skin grafts used as initial primary coverage at two weeks postburn for young patients (mean age between three and four years) for deep contact palm burns. When reviewed three years after burn, only three of 14 full-thickness grafted palms required secondary scar release compared with 10 of 16 split-thickness grafted palms. However, the two groups were not exactly comparable because the full-thickness skin grafted group had a mean $2 \%$ total body surface burn, while the patients who received STSGs had a mean $12 \%$ total body surface burn.

In our experience, the STSG for reconstruction has worked well in the first web in the grown hand when used in patients 11 years of age or older. However, this method has proved to be less successful when used for children under three years of age. Although split-thickness skin engraftment was adequate, the deformity gradually reappeared, which can be explained by one or more of wound contraction, difficulty fitting or wearing postoperative resting splints or a consequence of growth where the STSG did not expand as quickly as the hand grew. In young patients, a full-thickness graft appears to be more successful to maintain long term abduction gained.

Meyer et al (7) reviewed 29 first web space contractures following burns all closed with two- or four-flap Z-plasties or double opposing Z-plasties, none of which required skin grafts. They noted that first dorsal interosseous release was required more often for adults than for children.

Woolf and Broadbent (8) favoured the four-flap Z-plasty for web spaces as well as axillary contractures because, where single-limb Z-plasty is used when there is scarring adjacent to only one margin of the central limb (usually the dorsum of the hand) but not the other (volar surface), the scarred dorsal flap must be transposed to fit the palmar defect from which more pliable skin has been lifted, necessitating a tight closure. By creating the four-flap Z-plasty, the skin from the unscarred margin breaks up the scarred tissue in the middle of the Z-plasty.

Hirshowitz et al (9) have employed a five-flap Z-plasty, which is a derivative of the double opposing Z-plasty. The $\mathrm{V}$-shaped flap based on the palmar side resurfaces the centre of the cleft with interdigitating Z's on either side. The end result configuration is quite similar to the $\mathrm{V}$ - to M-plasty described subsequently by Alexander et al (10) used in the first web space as well as between the fingers. The Hirshowitz et al (9) technique employs the principle described by Mustarde (11) for medial epicanthal correction, but differs in that the epicanthal flap must be advanced, whereas in the hand the central V-flap is folded toward its raw side into the defect without advancement.

Browne et al (12) described a dorsally based rectangular flap for syndactyly of webs two, three and four. Housinger et al (13) incorporated this concept to create the goal postflap, which is a palmar-based rectangular flap that folds into a Y-shaped dorsal release. This introduces palmar skin into the dorsum similar to the principle of the fiveflap V to M advancement of Hirshowitz et al (9) and Alexander et al (10).

Sandzén (14) described a proximally based pedicle flap transposed from the dorsum of the thumb into the first web following contracture release for a dorsal hand burn previ- 
ously resurfaced with a flap that was transposed. The flap resembles that described by Spinner (15) raised from the dorsum of the index finger employed for Volkmann's ischemia contracture and congenital absence of the thumb. Borenstein et al (16) tissue expanded the dorsal skin of the hand. The expanded skin provided a proximally based flap advanced to resurface the released first web space.

The reversed radial forearm fascial flap covered with a free skin graft has been used by Jin et al (17) following first web burn scar release in 17 patients, many of whom also had dorsal scars of the wrist as well as MP joint hyperextension, but undamaged volar forearm skin. Cherup et al (18) used this flap lifted from a forearm that had previously had STSG applied to fascia.

Araico et al (19) required an abdominal flap to cover a buried bent $\mathrm{K}$-wire functioning as a permanent internal dynamic spring to separate the first and second metacarpals following abductor release. Miura (20) has used paired opposing abdominal flaps to resurface both sides of combined volar and dorsal first web space defects, while Bonola and Fiocchi (21) used the opposite upper arm for a double cross arm flap using opposing triangular flaps with the interposed donor arm abducting the thumb during the first stage of transfer. Scheker et al (22) have used the lateral arm fasciocutaneous free flap to resurface the first web with sensation provided by anatomosing the lower lateral cutaneous nerve of the flap to the cutaneous branch of the radial nerve.

\section{REFERENCES}

1. Snelling CFT. Delayed skin graft application following burn scar release of the face and hand. Ann Plast Surg 1983;10:349-58.

2. Littler JW. The prevention and the correction of adduction contracture of the thumb. Clin Orthop 1959;13:182-92.

3. Mutz SB. Thumb web contracture. Hand 1972;4:236-46.

4. Stern PJ, Neal HW, Carter W, MacMillan BG. Classification and management of burned thumb contractures in children. Burns 1985;11:168-74.

5. Pensler JM, Steward R, Lewis SR, Herndon DN. Reconstruction of the burned palm: full-thickness versus split-thickness skin grafts - long term follow-up. Plast Reconstr Surg 1988;81:46-9.

6. Schwanholt C, Greenhalgh DG, Warden GD. A comparison of full-thickness versus split-thickness autografts for the coverage of deep palm burns in the very young pediatric patient. J Burn Care Rehabil 1993;14:29-33.

7. Meyer RD, Gould JS, Nicholson B. Revision of the first web space: techniques and results. South Med J 1981;74:1204-8.

8. Woolf RM, Broadbent TR. The four-flap Z-plasty. Plast Reconstr Surg 1972;49:48-51.

9. Hirshowitz B, Karev A, Rousso M. Combined double Z-plasty and Y-V advancement for thumb web contracture. Hand 1975;7:291-3.

10. Alexander JW, MacMillan BG, Martel L. Correction of postburn syndactyly: an analysis of children with introduction of the VM-plasty and postoperative pressure inserts. Plast Reconstr Surg 1982;70:345-52.

11. Mustarde JC. The treatment of ptosis and epicanthal folds. Br J Plast Surg 1959;12:252-8.
The technique reviewed in this paper combines a standard volar and palmar release that creates a defect that is effectively covered by a medium-thickness STSG that can be readily and rapidly harvested. Abduction has been maintained in the hand that is at or close to adult size when released. Adequate soft-tissue release is the important first step and, where necessary, should include partial division of the first dorsal interosseous and thumb adductor. If indicated because of cupping, release of the palmar scar between the thenar and hypothenar eminence is done and may include a carpal tunnel release. The first web space release must accomplish both full radial and palmar abduction of the thumb. Split-thickness skin engraftment was not compromised by hematoma formation. With immediate grafting, the tourniquet was deflated and major bleeding stopped before the tourniquet was reinflated for graft application. The bolus dressing was anchored in place to supply maximum pressure before the tourniquet was deflated at end of the procedure. Postoperative compression gloves, which should include appropriate first web space inserts and night first web abduction splints for at least six months, are necessary.

ACKNOWLEDGEMENTS: The author thanks Maureen Bérard for preparation of the manuscript and the British Columbia Professional Fire Fighters Association for financial support for statistical analysis.
12. Browne EZ, Teague MA, Snyder CC. Burn syndactyly. Plast Reconstr Surg 1978;62:92-5.

13. Housinger TA, Ivers B, Warden GD. Release of the first web space with the "goalpost" procedure in pediatric burns. J Burn Care Rehabil 1993;14:353-5.

14. Sandzén SC. Dorsal pedicle flap for resurfacing a moderate thumb-index web contracture release. J Hand Surg 1982;7:21-4.

15. Spinner M. Fashioned transpositional flap for soft tissue adduction contracture of the thumb. Plast Reconstr Surg 1969;44:345-8.

16. Borenstein A, Yaffe B, Seidman DS, Engel J. Tissue expansion in reconstruction of postburn contracture of the first web space of the hand. Ann Plast Surg 1991;26:463-5.

17. Jin YT, Guan WX, Shi TM, Quian YL, Xu LG, Chang TS. Reversed island forearm fascial flap in hand surgery. Ann Plast Surg 1985;15:340-7.

18. Cherup LL, Zachary LS, Gottlieb LJ, Petti CA. The radial forearm skin graft-fascial flap. Plast Reconstr Surg 1990;85:898-902.

19. Araico J, Valdes JL, Oritz JM. An internal wire splint for adduction contracture of the thumb. Plast Reconstr Surg 1971;48:339-42.

20. Miura T. Use of paired abdominal flaps for release of adduction contractures of the thumb. Plast Reconstr Surg 1979;63:242-4.

21. Bonola A, Fiocchi R. Cross-arm double flap in the repair of severe adduction contracture of the thumb. Hand 1975;7:287-90.

22. Scheker LR, Lister GD, Wolff TW. The lateral arm free flap in releasing severe contracture of the first web space. J Hand Surg [Br] 1988;13:146-50. 